\title{
Independent prognostic value of coronary artery calcium score and coronary computed tomography angiography in an outpatient cohort of low to intermediate risk chest pain patients
}

\author{
M.J. Bom ${ }^{1} \cdot$ P.M. Van der Zee ${ }^{1}$ F.M. Van der Zant ${ }^{2} \cdot$ R.J.J. Knol ${ }^{2} \cdot$ J.H. Cornel ${ }^{1}$
}

Published online: 15 February 2016

(C) The Author(s) 2016. This article is published with open access at Springerlink.com

\begin{abstract}
Background Limited studies report on the additional prognostic value of coronary computed tomography angiography (CCTA) and the coronary artery calcium score (CACS). Methods For a median of 637 days, 1551 outpatients with chest pain, without known coronary artery disease (CAD) and low or intermediate pre-test probability of CAD, were followed for major adverse cardiac events (MACE), defined as death, myocardial infarction or late revascularisation. Cox proportional hazard regression was used to evaluate the independent prognostic value of CCTA and CACS.

Results MACE occurred in 23 patients (1.5\%): death (3, $0.2 \%)$, myocardial infarction $(4,0.3 \%)$ and late revascularisation (16, 1.3\%). Multivariate analysis showed an independent prognostic value of CCTA $(p<0.001)$, CACS of $100-400(p=0.035)$ and CACS of $>400(p=0.021)$. CCTA showed obstructive CAD in $3.1 \%$ of patients with $\mathrm{CACS}=0$. No events occurred in patients with CACS $=0$ without obstructive CAD at CCTA, whereas $2 / 23$ patients ( $9 \%)$ with CACS $=0$ with obstructive CAD had a MACE.

Conclusions Our study shows that both CCTA and higher CACS categories have independent prognostic value in chest pain patients with low to intermediate pre-test probability of obstructive CAD, in which CCTA is appropriate. Furthermore a non-negligible amount of patients with CACS $=0$ have obstructive CAD at CCTA. CCTA can be used in these patients to identify those at risk for MACE.
\end{abstract}

M.J. Bom

bommichiel@gmail.com; m.j.bom@mca.nl

Department of Cardiology, Medical Centre Alkmaar, Alkmaar, The Netherlands

2 Department of Nuclear Medicine, Medical Centre Alkmaar, Alkmaar, The Netherlands
Keywords CCTA · Coronary computed tomography angiography · Prognosis · CACS - Coronary artery calcium $\cdot$ MACE

\section{Introduction}

Quantification of coronary artery calcium by computed tomography represents a reliable estimate of atherosclerotic plaque burden and the prognostic value of the coronary artery calcium score (CACS) is well established [1, 2]. Recently, coronary computed tomographic angiography (CCTA) has emerged as an important imaging tool to detect the presence and extent of coronary artery disease (CAD) $[3,4]$. Several large prospective trials have demonstrated the prognostic value of CCTA, with a high negative predictive value for the occurrence of major adverse cardiac events (MACE) [5-11]. Overestimation of the severity of $\mathrm{CAD}$ in patients with a high pre-test probability is, however, a known limitation of CCTA $[12,13]$. Thus, the appropriate use criteria advise to only use CCTA in patients with low or intermediate pre-test probability [14]. The aim of this study was therefore to evaluate the independent prognostic value of CCTA and CACS in a routine clinical cohort of symptomatic patients with low or intermediate pre-test probability, in which CCTA is appropriate.

\section{Methods}

Population

From 13 December 2011 to 26 August 2014 all patients with chest pain with low or intermediate pre-test probability of $\mathrm{CAD}$, referred for CCTA from the outpatient clinic after a 


\title{
Advertisement placed here.
}

\author{
CSS Bohn

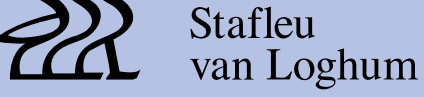 \\ Springer Media
}

Houten 2016 


\title{
Advertisement placed here.
}

\author{
CSS Bohn

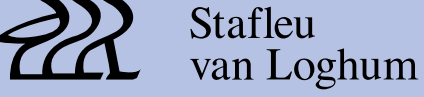 \\ Springer Media
}

Houten 2016 


\title{
Advertisement placed here.
}

\author{
CSS Bohn

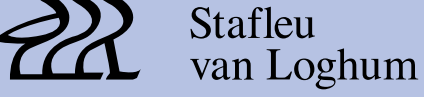 \\ Springer Media
}

Houten 2016 


\title{
Advertisement placed here.
}

\author{
CSS Bohn

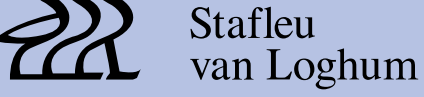 \\ Springer Media
}

Houten 2016 
diagnostic work up, were prospectively included. None of the patients had a prior history of CAD. The pre-test probability was calculated using the Duke Clinical Score [15]. A pre-test probability $<15 \%$ was defined as low and $15-85 \%$ was defined as intermediate, according to the ESC guidelines [3].

Baseline characteristics including age, gender, and cardiovascular risk factors were prospectively entered in the database. All patients gave written informed consent for usage of their data.

\section{Follow-up}

Patients were followed for MACE, defined as all-cause mortality, myocardial infarction or revascularisation (either CABG or PCI). A 60-day landmark was used to differentiate between CCTA-driven invasive coronary angiography and late revascularisation, which is considered to be indicative for the prognostic value of CCTA. Patients with referral for invasive coronary angiography in the outpatient setting within 60 days after CCTA and subsequent revascularisation were considered to be CCTA-driven and not MACE. All other revascularisations within follow-up were considered to be MACE. Information on myocardial infarction and revascularisation was obtained from the electronic medical records. Information on mortality was obtained from the municipal personal records database.

\section{CCTA preparation, acquisition and analysis}

Patient preparation, image acquisition, and image analysis were performed as previously described [16] and as briefly described below.

All scans were performed with a $2 \times 64$-slice flying focal spot, effectively $2 \times 128-$ slice (Somatom Definition Flash; Siemens Medical Systems, Erlangen, Germany) and were evaluated by a Certification Board of Cardiovascular Computed Tomography accredited nuclear medicine physician and a cardiologist experienced in the interpretation of CCTA in consensus. In case of disagreement a third opinion was decisive.

\section{Radiation dose}

The radiation dose delivered was generated automatically by the scanner software and represented as dose length product. The effective dose was calculated by multiplying the dose length product with the $\mathrm{k}$-factor of $0.014 \mathrm{mSv} \times(\mathrm{mGy} \times \mathrm{cm})^{-1}$, which is generally used in cardiac CT studies.

\section{Definition of CAD}

Obstructive CAD was defined as a lumen stenosis in any of the large vessels of $>50 \%$, either the left main artery, left anterior descending artery, circumflex artery or right coronary artery. Normal coronary arteries were defined as $\mathrm{CACS}=0$ and no coronary plaques. Non-obstructive CAD was defined as CACS $>0$ and/or any plaque that did not meet the criteria for obstructive CAD.

\section{Statistical analysis}

Statistical analysis was performed using SPSS software, version 22.0.0 (SPSS Inc, Chicago, Illinois). Continuous variables are presented as mean $\pm \mathrm{SD}$ and categorical variables as frequencies with percentages. Continuous variables were tested for normal distribution.

Kaplan-Meier analysis was used to assess MACE-free survival stratified by CACS and CCTA results. Univariate and subsequent multivariate Cox proportional hazard regression were used to evaluate the independent prognostic value of CACS and CCTA beyond clinical risk factors. The multivariate Cox regression was done in stepwise fashion according to the backwards approach, with $p<0.10$ as threshold for removal of variables. Clinical risk factors included in the univariate analysis were male gender, age, diabetes, smoking, hyperlipidaemia, hypertension, and family history of CAD. Selection of variables for entry in the multivariable Cox proportional hazard regression was based on univariate analysis with a threshold of $p<0.10$.

\section{Results}

\section{Population}

A total of 1560 patients were initially included in the database for follow-up. Follow-up could be obtained in $99.4 \%$ of the patients. Nine patients emigrated to a foreign country and were lost to follow-up. The total number of studied patients was 1551. An overview of the baseline characteristics is shown in Table 1.

\section{CCTA data}

CACS, CCTA results and radiation dose are summarised in Table 2. Two patients with left main CAD on CCTA also had three-vessel disease.

\section{Follow-up}

The cohort was followed for a median of 637 days. MACE occurred in 23 patients during follow-up. Three $(0.2 \%)$ 
Table 1 Baseline characteristics

\begin{tabular}{ll}
\hline Variable & Total $(n=1551)$ \\
\hline Demographics & $58.0 \pm 10.2$ \\
Age & $968(62.4 \%)$ \\
Women & $26.6 \pm 4.5$ \\
Body mass index & $121(7.8 \%)$ \\
Diabetes & $6.8 \pm 1.6$ \\
Hbalc $(n=53)^{\mathrm{a}}$ & $464(29.9 \%)$ \\
Hypertension & $393(25.3 \%)$ \\
Hyperlipidaemia & $731(47.2 \%)$ \\
Family history of CAD & \\
Smoking & $277(17.9 \%)$ \\
eGFR $<60$ & $44(2.8 \%)$ \\
Duke clinical score & \\
-Low $(<15 \%)$ & $527(34.0 \%)$ \\
-Low-intermediate $(15-50 \%)$ & $760(49.0 \%)$ \\
-High-intermediate $(50-85 \%)$ & $264(17.0 \%)$ \\
Baseline medication use & \\
Aspirin & $499(32.2 \%)$ \\
Statin & $531(34.2 \%)$ \\
Beta-blocker & $604(38.9 \%)$ \\
ACE-i/ARB & $390(25.1 \%)$ \\
Calcium channel blocker & $96(6.2 \%)$ \\
Nitrate & $49(3.2 \%)$ \\
Acenocoumarol & $38(2.5 \%)$ \\
\hline
\end{tabular}

eGFR estimated glomerular filtration rate, $A C E-I / A R B$ ACEinhibitor/angiotensin receptor blocker.

${ }^{\mathrm{a}} \mathrm{Hba1C}$ was documented in only 53 patients.

${ }^{b}$ In 2 patients family history of CAD was missing.

patients died, $4(0.3 \%)$ patients had a myocardial infarction, and $20(1.3 \%)$ patients had non-CCTA-driven revascularisations. Four patients $(0.3 \%)$ were acute revascularisations in myocardial infarction and $16(1.0 \%)$ were late revascularisations, referred beyond 60 days after CCTA because of ongoing symptoms.

Figure 1 shows the MACE-free survival estimates stratified by CACS and CCTA. Both increase in CACS and presence of obstructive CAD at CCTA were associated with decreased MACE-free survival (log-rank $p$-value $<0.01$ ).

MACE occurred in no patients with normal coronary arteries at CCTA, in $1.0 \%$ of patients with non-obstructive CAD at CCTA and in $7.5 \%$ of patients with obstructive CAD at CCTA. During follow-up, MACE occurred in $0.3 \%$ of patients with $\mathrm{CACS}=0$, in $1.0 \%$ of patients with CACS $1-100$, in $4.2 \%$ with CACS $101-400$ and in $7.1 \%$ with $\mathrm{CACS}>400$.

Of all 739 patients with $\mathrm{CACS}=0,23$ (3.1\%) had obstructive CAD at CCTA and $62(8.4 \%)$ had non-obstructive CAD at CCTA. No events occurred in patients with $\mathrm{CACS}=0$ and no obstructive CAD at CCTA, whereas $2 / 23$ patients $(9 \%)$ with $\mathrm{CACS}=0$ and obstructive $\mathrm{CAD}$ at CCTA had a MACE during follow-up: late revascularisation in both cases. MACE-free survival was significantly
Table 2 CCTA data

\begin{tabular}{ll}
\hline Variable & Total $(n=1551)$ \\
\hline Coronary artery calcium score & $739(47.6 \%)$ \\
$0.1-100$ & $498(32.1 \%)$ \\
$100-400$ & $215(13.9 \%)$ \\
$>400$ & $99(6.5 \%)$ \\
CCTA results & \\
Normal coronary arteries & $654(42.2 \%)$ \\
Non-obstructive CAD & $683(44.0 \%)$ \\
Obstructive CAD $(>50 \%)$ & $214(13.8 \%)$ \\
1-vessel & $164(10.6 \%)$ \\
2-vessel & $34(2.2 \%)$ \\
3-vessel & $13(0.8 \%)$ \\
Left main & $4(0.3 \%)$ \\
High-risk lesions ${ }^{\mathrm{a}}$ & $48(3.1 \%)$ \\
Radiation dose & \\
All patients $(n=1551)$ & $2.4 \pm 2.0 \mathrm{mSv}$ \\
High-pitch FLASH scans $(n=1130)$ & $1.6 \pm 0.7 \mathrm{mSv}$ \\
Prospectively triggered scans $(n=386)$ & $4.5 \pm 2.5 \mathrm{mSv}$ \\
Retrospectively triggered scans $(n=35)$ & $6.5 \pm 2.7 \mathrm{mSv}$ \\
\hline$C C T A$ coronary computed tomography angiography, CAD coronary \\
artery disease, $P T P$ pre-test probability. \\
ahigh-risk lesions were defined as left main, three-vessel and/or \\
proximal left anterior descending disease.
\end{tabular}

worse in CACS 0 patients with obstructive CAD, log-rank p-value $<0.001$.

\section{Independent prognostic value of CACS and CCTA}

The evaluation of the predictive value of CACS and CCTA by univariate and multivariate Cox proportional hazard regression is shown in Table 3. By univariate analysis, increased risk of MACE was observed with increasing CACS (p-value $<0.001$ ). Furthermore, obstructive CAD at CCTA was associated with a 13 -fold increase in unadjusted risk of MACE. Of all clinical risk factors, male gender and hypertension had a $p$-value of $<0.10$ and were subsequently included in the multivariate analysis.

In the multivariate model, obstructive disease on CCTA was independently associated with increased risk of MACE $(p<0.001)$. Furthermore, CACS of $100-400(p=0.035)$ and CACS of $>400(0.021)$ were independent predictors for the occurrence of MACE, whereas a CACS of $0-100(p=0.29)$ had no independent predictive value for the occurrence of MACE.

\section{Discussion}

Our study shows that in a routine clinical cohort of patients referred from the outpatient clinic with chest pain with low to intermediate pre-test probability of obstructive $\mathrm{CAD}$, the 

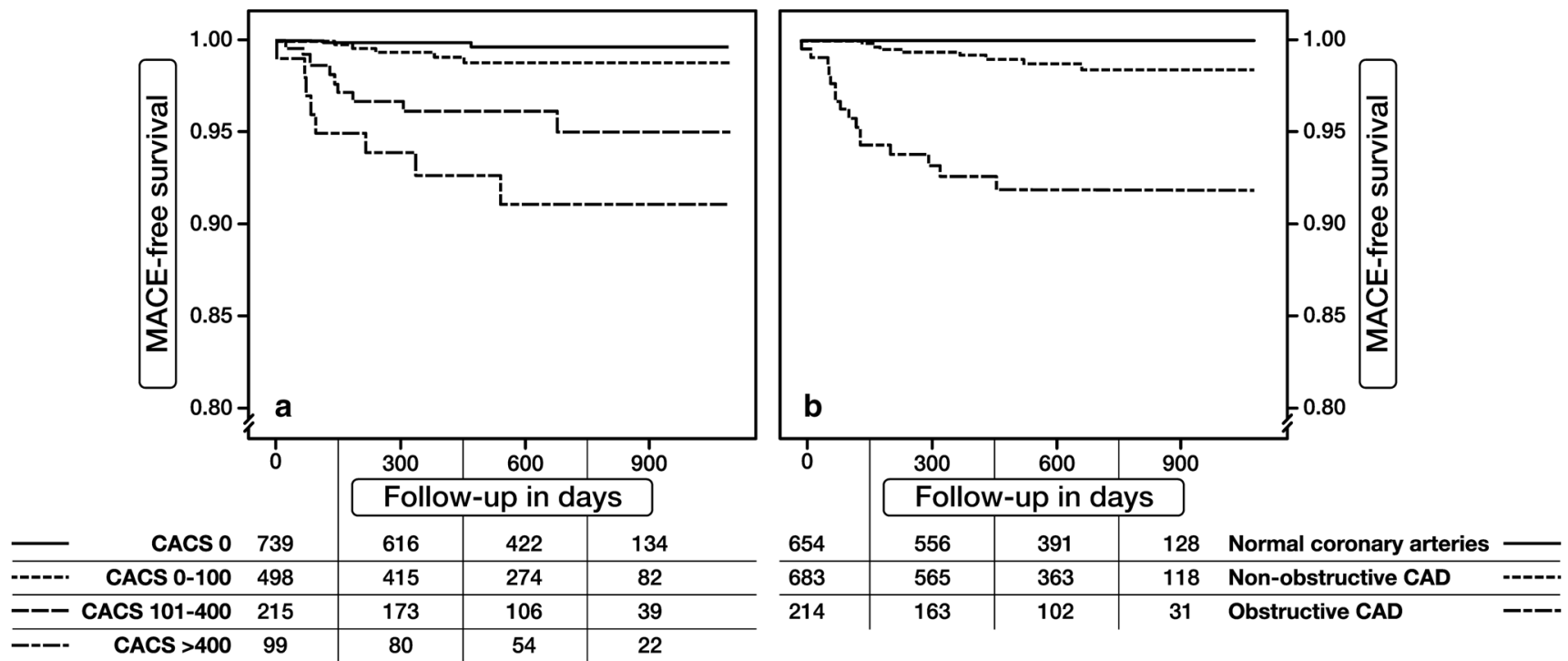

Fig. 1 Kaplan-Meier curves of MACE-free survival stratified by CACS (a) and CCTA results (b)

Table 3 Univariate and multivariate Cox regression of risk factors, CACS and CCTA

\begin{tabular}{|c|c|c|c|}
\hline Variable & HR & $95 \% \mathrm{CI}$ & $P$-value \\
\hline \multicolumn{4}{|l|}{ Univariate analysis } \\
\hline Male gender & 2.18 & $0.96-4.97$ & $0.064^{\mathrm{a}}$ \\
\hline Age & 1.02 & $0.98-1.07$ & 0.26 \\
\hline Diabetes mellitus & 1.15 & $0.55-2.43$ & 0.71 \\
\hline Hypertension & 2.31 & $1.02-5.24$ & $0.045^{\mathrm{a}}$ \\
\hline Family history & 1.76 & $0.76-4.07$ & 0.19 \\
\hline Smoking & 0.71 & $0.21-2.40$ & 0.58 \\
\hline Hyperlipidaemia & 1.97 & $0.85-4.55$ & 0.11 \\
\hline \multicolumn{4}{|l|}{ CACS } \\
\hline $\mathrm{CACS}=0$ & Reference & Reference & - \\
\hline CACS $0-100$ & 3.71 & $0.72-19.12$ & 0.12 \\
\hline CACS $100-400$ & 15.82 & $3.42-72.23$ & $<0.001^{\mathrm{a}}$ \\
\hline $\mathrm{CACS}>400$ & 26.81 & $5.57-129.09$ & $<0.001^{\mathrm{a}}$ \\
\hline Obstructive CAD at CCTA & 15.28 & $6.28-37.14$ & $<0.001^{\mathrm{a}}$ \\
\hline \multicolumn{4}{|l|}{ Multivariate analysis } \\
\hline Obstructive CAD at CCTA & 7.03 & $2.57-19.22$ & $<0.001$ \\
\hline \multicolumn{4}{|l|}{ CACS } \\
\hline $\mathrm{CACS}=0$ & Reference & Reference & - \\
\hline CACS $0-100$ & 2.46 & $0.46-13.14$ & 0.29 \\
\hline CACS $100-400$ & 5.97 & $1.14-31.31$ & 0.035 \\
\hline CACS $>400$ & 7.72 & $1.37-43.55$ & 0.021 \\
\hline
\end{tabular}

$C A C S$ coronary artery calcium score, CCTA coronary computed tomography angiography, $H R$ hazard ratio

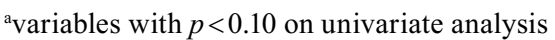

prognosis of a CACS of 0 is excellent, with MACE during follow-up in only $0.3 \%$ of patients. However, a non-negligible amount of patients with $\mathrm{CACS}=0$ had obstructive CAD (3\%). Presence of CAD in patients with $\mathrm{CACS}=0$ was associated with worse prognosis.
Furthermore, our study shows that although overall prognosis in this clinical cohort was excellent, presence of obstructive CAD on CCTA had an independent prognostic value. CACS of 0-100 did not show independent prognostic value of CCTA; however CACS 100-400 and >400 did show independent prognostic value.

\section{Prognostic value of CACS and CCTA}

The prognostic value of CACS has been previously demonstrated in large meta-analyses [1,2]. A recently published large multicentre international cohort by Al-Mallah et al. reported a gradual increase in event rates in patients with CACS $=0$, CACS 1-100, 100-399 and >400, with comparable event rates to our study [10].

Multiple studies have previously reported on the prognostic value of CCTA for the occurrence of adverse events [6-11]. Our study results are in line with a recently published meta-analysis of 41,960 patients which reported that normal coronary arteries at CCTA are associated with a very low annual event rate of $<0.5 \%$ and that obstructive CAD at CCTA is associated with higher event rates of $12.5 \%$ [17]. The slightly lower event rate in patients with obstructive CAD at CCTA in our study might be explained by the fact that no patients with a high pre-test probability were included.

\section{CACS of 0}

Our study, in accordance with prior studies, showed an excellent prognosis of symptomatic patients with $\mathrm{CACS}=0$, with an event rate $<1 \%$ within 2 years of fol- 
low-up [10, 18-20]. Because of higher costs, the need to administer intravenous contrast and the higher radiation burden of CCTA, it remains the subject of discussion whether one should proceed with CCTA after CACS $=0$ in all patients, given the excellent prognosis of these patients. The ESC guidelines on stable CAD do not recommend the use of CACS to identify individuals at risk [3]. However, a recent update of the American College of Cardiology and the American Heart Association stated that the exclusion of coronary calcium by CACS may be reasonable before considering further testing in symptomatic patients with low to intermediate pre-test probability [21]. Our study confirms findings of recent studies that in symptomatic patients with $\mathrm{CACS}=0$, a non-negligible $1.4-4.5 \%$ have evidence of obstructive CAD at CCTA $[10,18,19]$. No events occurred during follow-up in patients with $\mathrm{CACS}=0$ and no obstructive CAD at CCTA in our study and obstructive CAD at CCTA was significantly associated with a worse prognosis. CCTA was thus able to accurately identify those with $\mathrm{CACS}=0$ at risk for future events. Performing CCTA after CACS $=0$ may still be advisable in symptomatic patients with low to intermediate pre-test probability to identify those at risk for future events.

\section{Independent prognostic value of CCTA}

Several studies have been published on the independent prognostic value of CCTA to CACS. Cho et al. reported that in 7590 studied asymptomatic patients, CCTA did not have prognostic value independent of CACS [22]. Other studies have shown that in predominantly symptomatic patients, CCTA has added prognostic value to CACS and clinical risk factors $[6,10,23]$. These studies, however, differ in study populations from our study. Hadamitzky et al. reported on the added prognostic value of CCTA to CACS in a population of both symptomatic and asymptomatic patients [23]. The single-centre study by Hou et al. included a substantial number of asymptomatic patients and patients with high pre-test probability [6]. Both Al-Mallah et al. and Van Werkhoven et al. studied solely symptomatic patients; $10 \%$ of their population, however, had a high pre-test probability [8, 10]. The appropriate use criteria advise to only use CCTA with a low or intermediate pre-test probability [14]. While our findings are mainly of a confirmatory nature, our study does provide some additional information about the independent prognostic value of CCTA to CACS in a Dutch routine clinical cohort of symptomatic patients, in which CCTA is appropriate [14].

\section{Independent prognostic value of CACS}

Data on the independent prognostic value of CACS in the CCTA era are limited [24-26]. Chaikriangkrai et al. recently reported independent prognostic value of both CCTA and CACS, with prognostic value across all categories of CACS [24]. In our study CACS had independent prognostic value, although only in the categories CACS $100-400$ and $>400$, whereas CACS 0-100 did not have prognostic value. This may be partly explained by the relatively low event rate and the lower risk population compared with the study population of Chaikriangkrai et al. The independent prognostic power of higher categories of CACS together with the fact that severe coronary calcification is associated with decreased diagnostic accuracy of CCTA, [27] support the use of CACS with CCTA as compared with CCTA only.

\section{Clinical implications}

The recently published PROMISE trial investigated symptomatic patients with suspected CAD who require noninvasive testing and reported no difference in outcome between an initial strategy of CCTA and functional testing [28]. However, questions were raised about the safety of the CCTA strategy because of a relatively high radiation dose. The reported radiation dose in previous reports on the prognostic value of CCTA ranged from 3 to $18 \mathrm{mSv}[5,23$, $28]$. With the use of a high-resolution $(2 \times 128)$ scanner and predominantly a flash or prospective scanning protocol, we were able to perform a complete CCTA (calcium score and angiography) with an excellent prognostic value and a substantially lower radiation burden of $2.4 \pm 2.0 \mathrm{mSv}$. Although the event rate was relatively low in our study, this further supports the use of an initial CCTA strategy in patients with low to intermediate pre-test probability of obstructive CAD.

\section{Study limitations}

The single-centre design of our study allows the evaluation of prognosis in a 'real world' clinical setting. However, our results may not be applied to all outpatient chest pain populations, i.e. asymptomatic or high-risk patients.

As in previous studies, a 60-day landmark was used to differentiate between CCTA-driven invasive coronary angiography and long-term revascularisation, which is considered to be indicative for the prognostic value of CCTA [6]. Recently, several studies have reported that CCTA has a significant effect on downstream patient management $[29,30]$. In our study 93 (6.0\%) patients underwent CCTA-guided revascularisation (referral for $\mathrm{PCI} / \mathrm{CABG}$ within 60 days of CCTA). Since the aim of our study was to evaluate the prognostic value of CCTA in a routine clinical cohort, these patients were not excluded for analysis. However, outcome of the patients might have been confounded by CCTAguided management changes. 


\section{Conclusion}

This study shows that both CCTA and CACS of 100-400 and $>400$ have an independent prognostic value for the occurrence of MACE in a routine clinical cohort of patients presenting to the outpatient clinic with atypical chest pain with low to intermediate pre-test probability of obstructive CAD, in which CCTA is appropriate. Furthermore our study shows that a non-negligible amount of patients with CACS $=0$ have obstructive CAD at CCTA. CCTA can be used in patients with $\mathrm{CACS}=0$ to identify those at risk for MACE.

Funding This work was not supported by any funding.

\section{Conflict of interests None declared.}

Open Access This article is distributed under the terms of the Creative Commons Attribution 4.0 International License (http://creativecommons.org/licenses/by/4.0/), which permits unrestricted use, distribution, and reproduction in any medium, provided you give appropriate credit to the original author(s) and the source, provide a link to the Creative Commons license, and indicate if changes were made.

\section{References}

1. Bellasi A, Lacey C, Taylor AJ, et al. Comparison of prognostic usefulness of coronary artery calcium in men versus women (results from a meta- and pooled analysis estimating all-cause mortality and coronary heart disease death or myocardial infarction). Am J Cardiol. 2007;100:409-14.

2. Budoff MJ, Shaw LJ, Liu ST, et al. Long-term prognosis associated with coronary calcification: observations from a registry of 25,253 patients. J Am Coll Cardiol. 2007;49:1860-70.

3. Task Force Members, Montalescot G, Sechtem U, et al. ESC guidelines on the management of stable coronary artery disease: the Task Force on the management of stable coronary artery disease of the European Society of Cardiology. Eur Heart J 2013;34:2949-3003.

4. Wall EE van der. Crown years for non-invasive cardiovascular imaging. (Part IV): 30 years of cardiac computed tomography. Neth Heart J. 2013;21:315-8.

5. Min JK, Dunning A, Lin FY, et al. Age- and sex-related differences in all-cause mortality risk based on coronary computed tomography angiography findings results from the International Multicenter CONFIRM (Coronary CT Angiography Evaluation for Clinical Outcomes: an International Multicenter Registry) of 23,854 patients without known coronary artery disease. J Am Coll Cardiol. 2011;58:849-60.

6. Hou ZH, Lu B, Gao Y, et al. Prognostic value of coronary CT angiography and calcium score for major adverse cardiac events in outpatients. JACC Cardiovasc Imaging. 2012;5:990-9.

7. Hadamitzky M, Taubert S, Deseive S, et al. Prognostic value of coronary computed tomography angiography during 5 years of follow-up in patients with suspected coronary artery disease. Eur Heart J. 2013;34:3277-85.
8. Werkhoven JM van, Schuijf JD, Gaemperli O, et al. Incremental prognostic value of multi-slice computed tomography coronary angiography over coronary artery calcium scoring in patients with suspected coronary artery disease. Eur Heart J. 2009;30:2622-9.

9. Graaf FR de, Velzen JE van, Boer SM de, et al. Non-invasive computed tomography coronary angiography as a gatekeeper for invasive coronary angiography. Int J Cardiovasc Imaging. 2013;29:221-8.

10. Al-Mallah MH, Qureshi W, Lin FY, et al. Does coronary CT angiography improve risk stratification over coronary calcium scoring in symptomatic patients with suspected coronary artery disease? Results from the prospective multicenter international CONFIRM registry. Eur Heart J Cardiovasc Imaging. 2014;15:267-74.

11. Werkhoven JM van, Gaemperli O, Schuijf JD, et al. Multislice computed tomography coronary angiography for risk stratification in patients with an intermediate pretest likelihood. Heart. 2009;95:1607-11.

12. Budoff MJ, Dowe D, Jollis JG, et al. Diagnostic performance of 64-multidetector row coronary computed tomographic angiography for evaluation of coronary artery stenosis in individuals without known coronary artery disease: results from the prospective multicenter ACCURACY (Assessment by Coronary Computed Tomographic Angiography of Individuals Undergoing Invasive Coronary Angiography) trial. J Am Coll Cardiol. 2008;52:1724-32.

13. Meijboom WB, Mieghem CA van, Mollet NR, et al. 64-Slice Computed Tomography Coronary Angiography in Patients with High, Intermediate, Or Low Pretest Probability of Significant Coronary Artery Disease. J Am Coll Cardiol. 2007;50:1469-75.

14. Taylor AJ, Cerqueira M, Hodgson JM, et al. ACCF/SCCT/ACR/ AHA/ASE/ASNC/NASCI/SCAI/SCMR 2010 appropriate use criteria for cardiac computed tomography. A report of the American College of Cardiology Foundation Appropriate Use Criteria Task Force, the Society of Cardiovascular Computed Tomography, the American College of Radiology, the American Heart Association, the American Society of Echocardiography, the American Society of Nuclear Cardiology, the North American Society for Cardiovascular Imaging, the Society for Cardiovascular Angiography and Interventions, and the Society for Cardiovascular Magnetic Resonance. J Am Coll Cardiol. 2010;56:1864-94.

15. Bayliss J. Duke Clinical Score: prediction of Coronary Heart Disease in a Patient with Chest Pain. 2009. http://www.zunis.org/ Duke\%20Chest\%20Pain\%20-\%20CAD\%20Predictor.htm.

16. Krul MM, Bogaard K, Knol RJ, et al. Coronary artery disease in patients with atypical chest pain with and without diabetes mellitus assessed with coronary CT angiography. BMJ Open Diabetes Res Care. 2014;2:e00004.

17. Habib PJ, Green J, Butterfield RC, et al. Association of cardiac events with coronary artery disease detected by 64 -slice or greater coronary CT angiography: a systematic review and meta-analysis. Int J Cardiol. 2013;169:112-20.

18. Villines TC, Hulten EA, Shaw LJ, et al. Prevalence and severity of coronary artery disease and adverse events among symptomatic patients with coronary artery calcification scores of zero undergoing coronary computed tomography angiography: results from the CONFIRM (Coronary CT Angiography Evaluation for Clinical Outcomes: an International Multicenter) registry. J Am Coll Cardiol. 2011;58:2533-40.

19. Kim YJ, Hur J, Lee HJ, et al. Meaning of zero coronary calcium score in symptomatic patients referred for coronary computed tomographic angiography. Eur Heart J Cardiovasc Imaging. 2012;13:776-85. 
20. Rijlaarsdam-Hermsen D, Kuijpers D, Dijkman PR van. Diagnostic and prognostic value of absence of coronary artery calcification in patients with stable chest symptoms. Neth Heart J. 2011;19:223-8.

21. Greenland P, Bonow RO, Brundage BH, et al. ACCF/AHA 2007 clinical expert consensus document on coronary artery calcium scoring by computed tomography in global cardiovascular risk assessment and in evaluation of patients with chest pain: a report of the American College of Cardiology Foundation Clinical Expert Consensus Task Force (ACCF/AHA Writing Committee to Update the 2000 Expert Consensus Document on Electron Beam Computed Tomography) developed in collaboration with the Society of Atherosclerosis Imaging. and Prevention and the Society of Cardiovascular Computed Tomography. J Am Coll Cardiol. 2007;49:378-402.

22. Cho I, Chang HJ, Sung JM, et al. Coronary computed tomographic angiography and risk of all-cause mortality and nonfatal myocardial infarction in subjects without chest pain syndrome from the CONFIRM Registry (coronary CT angiography evaluation for clinical outcomes: an international multicenter registry). Circulation. 2012;126:304-13.

23. Hadamitzky M, Distler R, Meyer T, et al. Prognostic value of coronary computed tomographic angiography in comparison with calcium scoring and clinical risk scores. Circ Cardiovasc Imaging. 2011;4:16-23.

24. Chaikriangkrai K, Velankar P, Schutt R, et al. Additive prognostic value of coronary artery calcium score over coronary computed tomographic angiography stenosis assessment in symptomatic patients without known coronary artery disease. Am J Cardiol. 2015;115:738-44.
25. Kwon SW, Kim YJ, Shim J, et al. Coronary artery calcium scoring does not add prognostic value to standard 64-section CT angiography protocol in low-risk patients suspected of having coronary artery disease. Radiology. 2011;259:92-9.

26. Hulten E, Bittencourt MS, Ghoshhajra B, et al. Incremental prognostic value of coronary artery calcium score versus $\mathrm{CT}$ angiography among symptomatic patients without known coronary artery disease. Atherosclerosis. 2014;233:190-5.

27. Abdulla J, Pedersen KS, Budoff M, Kofoed KF. Influence of coronary calcification on the diagnostic accuracy of 64-slice computed tomography coronary angiography: a systematic review and metaanalysis. Int J Cardiovasc Imaging. 2012;28:943-53.

28. Douglas PS, Hoffmann U, Patel MR, et al. Outcomes of anatomical versus functional testing for coronary artery disease. N Engl J Med. 2015;372:1291-300.

29. Cheezum MK, Hulten EA, Smith RM, et al. Changes in preventive medical therapies and $\mathrm{CV}$ risk factors after CT angiography. JACC Cardiovasc Imaging. 2013;6:574-81.

30. Hulten E, Bittencourt MS, Singh A, et al. Coronary artery disease detected by coronary computed tomographic angiography is associated with intensification of preventive medical therapy and lower low-density lipoprotein cholesterol. Circ Cardiovasc Imaging. 2014;7:629-38.

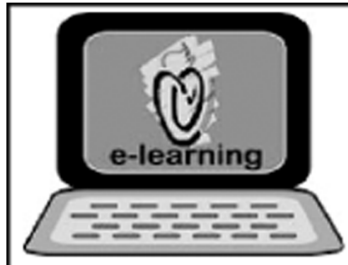

\section{CVOI E-learning formula!}

This is the CVOI e-learning article. The author has prepared 10 questions which are available through the website of the Cardiovascular Educational Institute (CVOI). Please follow the instructions below.

After finishing the questions you will be asked to fill in your name, hospital and e-mail address; then press the button 'verzenden'.

When 6 out of the 10 questions are answered correctly, you acquire 1 accreditation point granted by the Quality Committee of the Netherlands Society of Cardiology (NVVC). The acquired point will be credited to your personal file in the GAIA system. You will also receive an e-mail with all the correct answers.

Over a period of one year 10 e-learning articles will appear in 10 subsequent NHJ editions. In each edition the e-learning article will be recognisable by a special icon. On an annual basis you can collect 10 accreditation points. The accreditation points are credited in the GAIA system by the CVOI.

If you need additional information, please contact the CVOI by e-mail: cvoi@cvoi.org or by phone: 030-2345001.

E.E. van der Wall

Chief editor NHJ
K.B. Schick

Coordinator CVOI 